\title{
ESTRUCTURA COMUNITARIA DEL ZOOPLANCTON ASOCIADA CON EL FITOPLANCTON Y LAS MASAS DE AGUA DEL ESTRECHO DE BRANSFIELD Y LA ISLA ELEFANTE DURANTE EL VERANO AUSTRAL DEL 2006
}

\section{ZOOPLANKTON COMMUNITY STRUCTURE AND ITS ASSOCIATION WITH THE PHYTOPLANKTON AND WATER MASSES FROM THE BRANSFIELD STRAIT AND ELEPHANT ISLAND DURING 2006 AUSTRAL SUMMER}

\author{
Jessica Bonicelli P. ${ }^{1}$, Diana López P. ${ }^{2}$, Noemí Ochoa L. ${ }^{3}$ y Rachael S. Shreeve ${ }^{4}$
}

Resumen

Durante el verano austral del año 2006, la abundancia y distribución espacial del zooplancton y su asociación con algunas variables físicas (masas de agua) y biológicas (fitoplancton) fueron investigadas en el Estrecho de Bransfield y al sureste de la Isla Elefante. La comunidad estuvo compuesta principalmente por copépodos, siendo las especies más abundantes Metridia gerlachei y Ctenocalanus sp. Mediante el análisis cluster se diferenciaron 3 grupos de estaciones asociados con las masas de agua. Un grupo se halló al sureste de la Isla Elefante y del Estrecho de Bransfield, donde predominó la masa de agua del mar de Weddell. Allí el fitoplancton fue escaso, mientras que el zooplancton fue abundante. Esto puede ser un ejemplo de un control top-down debido al pastoreo. Otro grupo se distribuyó al noroeste del Estrecho, donde predominaron las aguas del mar de Bellingshausen, en esta zona el zooplancton tuvo abundancias bajas, mientras que el fitoplancton fue encontrado en altas concentraciones. El último grupo fue encontrado en el centro del estrecho, en donde se encuentra el Frente de Bransfield. A pesar de las altas abundancias de fitoplancton, la mayoría de especies zooplanctónicas presentaron bajas abundancias, pudiendo ser por las fuertes turbulencias que se forman en el Frente. Sin embargo, Metridia gerlachei fue la más abundante en esta zona probablemente por su extensa migración vertical.

Palabras clave: Estructura comunitaria, zooplancton, fitoplancton, Antártida, Estrecho de Bransfield, Isla Elefante, Frente de Bransfield

\begin{abstract}
During the austral summer of 2006 the spatial distribution of zooplankton and its association with some abiotic (water masses) and biotic variables (phytoplankton) were investigated in the Bransfield Strait and to the southeast of Elephant Island, Southern Ocean. The zooplankton community was mainly composed of copepods; the main species present were Metridia gerlachei and Ctenocalanus sp. Nearest neighbor cluster analysis of zooplankton indicated the presence of three major station groupings which were influenced by water masses. One group was found in the southeast of the Bransfield Strait and Elephant Island, where the Weddell water masses predominated. This showed low phytoplankton abundance, whilst zooplankton abundance was high. This may be an example of top down grazing control. A second group was found in the northwest of the Strait, where the Bellingshausen water masses predominated. Here the zooplankton abundance was low, whilst the phytoplankton was found in high concentrations. A third group was found in the Bransfield front. Despite the high abundances of phytoplankton located here, zooplankton abundance was low. This could be due to the strong turbulence at the front. However, Metridia gerlachei was found in much higher concentrations here than other copepods. This species may be able to cope with such conditions due to its extensive diurnal vertical migrations.
\end{abstract}

Key words: Community structure, zooplankton, phytoplankton, Antarctic, Bransfield Strait, Elephant Island, Bransfield Front

\section{Introducción}

El estrecho de Bransfield esta ubicado entre la Península Antártica y el archipiélago de las Islas Shetland del sur. Esta zona es una región muy dinámica donde se encuentran las masas de agua del mar de Bellingshausen del noroeste y del mar de Weddell del sureste (Tokarczyk, 1987; Niiler et al., 1991; García et al., 1994; López et al., 1999), 
formando un frente entre ellas llamado el frente de Bransfield (Figueiras et al., 1998; García et al., 2002). Asimismo es considerada por muchos autores como una zona muy productiva para todos los niveles tróficos (Huntley et al., 1990; Brinton, 1991; Zhou et al., 1994).

El zooplancton es uno de los eslabones más importantes dentro del ecosistema marino antártico, ya que forma parte de los consumidores primarios y sirven de alimento principalmente a aves, mamíferos y peces pelágicos (Hubold \& Ekau, 1990; Bargu et al., 2002; Takahashi et al., 2003). También se ha demostrado que Euphausia superba Dana 1852 ("krill"), especie muy importante por servir de alimento para muchos de los grandes carnívoros (Quetin \& Ross, 1991), se alimenta de otras especies zooplanctónicas como algunos copépodos (Atkinson \& Zinder, 1997)

Se han realizados muchos estudios que señalan que la competencia, la depredación y la disponibilidad del alimento son factores que determinan la abundancia y la distribución del zooplancton (Ward et al., 2005; Atkinson et al., 1999). Otro factor relevante que afecta el comportamiento del zooplancton es la hidrografía de las masas de agua. En el norte de la isla Georgia del Sur, las diferentes características hidrográficas de la región influyen en la estructura comunitaria del zooplancton (Ward et al., 2005). Asimismo mediante estudios realizados por Silva et al. (1995) alrededor de la Isla Elefante, se han obtenido diferentes regiones con características físicas y químicas propias que influyen en la producción primaria, lo cual puede afectar a la comunidad zooplanctónica.

Este estudio es una continuación de las diversas expediciones al estrecho de Bransfield en las que el Instituto del Mar del Perú (IMARPE) ha participado desde 1988, y tiene como objetivo principal conocer la estructura comunitaria del zooplancton y su relación con el fitoplancton y las masas de agua en el estrecho de Bransfield y al sureste de la Isla Elefante durante el verano austral del año 2006.

\section{Materiales y métodos}

Área de estudio.

El área de estudio comprende el Estrecho de Bransfield y el sureste de la Isla Elefante $\left(62^{\circ} 17.28^{\prime} \mathrm{S}\right.$, $\left.58^{\circ} 19.58^{\prime} \mathrm{W} ; 6^{\circ} 14.49^{\prime} \mathrm{S}, 54^{\circ} 38.16^{\prime} \mathrm{W}\right)$ (Figura 1). Obtención y análisis de las muestras de zooplancton

Se colectó un total de 22 muestras de zooplancton en las 11 estaciones de muestreo (Figura 1), entre el 6 y el 14 de Enero del 2006 en el crucero 0601 BIC/HUMBOLDT. Se utilizó una red Bongo con mallas de 300 y 500 micras. Los arrastres fueron oblicuos a una velocidad de 3 nudos desde los $200 \mathrm{~m}$ de profundidad hasta la superficie. Las muestras fueron fijadas y preservadas con formaldehído al $2 \%$.

Para el análisis se emplearon las muestras de la malla de 300 micras (11 muestras). Los datos de abundancia se expresaron en ind/1000 $\mathrm{m}^{3}$. El análisis se llevo a cabo en el laboratorio de Ecología Acuática de la Universidad Nacional Mayor de San Marcos (UNMSM). En lo posible los organismos se determinaron a nivel de especie.

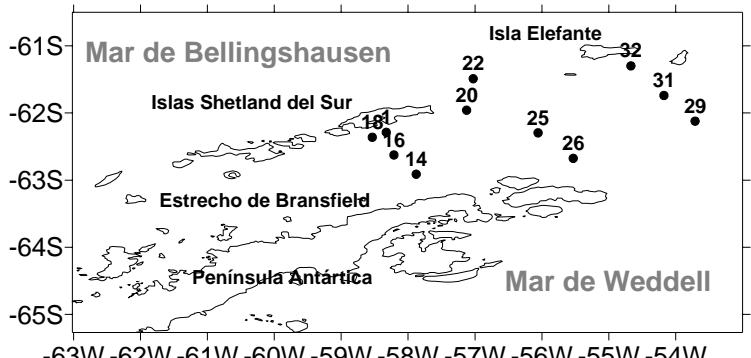

$-63 W-62 W-61 W-60 W-59 W-58 W-57 W-56 W-55 W-54 W$

Figura 1. Distribución de las estaciones de muestreo en la zona de estudio.

Obtención y análisis de las muestras de fitoplancton

Las muestras de fitoplancton se colectaron en 10 estaciones. Estas fueron tomadas con botellas Niskin a profundidades de $0,10,20,30,40,50,70$ y $100 \mathrm{~m}$. Luego se analizaron cualitativa y cuantitativamente en el laboratorio de Ecología Acuática - UNMSM, según la técnica de Utermöhl. Se sedimento $50 \mathrm{~mL}$ de agua, los organismos nanoplanctonicos se contaron en un área correspondiente a $2 \mathrm{~mL}$, con un aumento de $400 \mathrm{x}$ y el resto se contó en toda la base de la cámara de sedimentación a menor aumento (200x). Las abundancias fueron integradas para obtener la abundancia en la columna de agua de $0-100$ metros. Los datos se expresaron en cel/L.

Los datos de temperatura y salinidad, obtenidos mediante un CTD (Conductivity/Temperature/Depth), fueron proporcionados por el Área de Oceanografía Física del IMARPE.

Análisis y procesamiento de los datos

El programa Primer 5 se utilizó para los análisis comunitarios. Los datos de abundancia fueron transformados a $\log (\mathrm{x}+1)$ y se uso el índice de BrayCurtis para los análisis de similaridad y de conglomerados (Cluster). Se realizó el análisis ANOSIM, para ver si hubo diferencias significativas o no entre los grupos de estaciones de muestreo, obtenidos mediante el análisis Cluster, con respecto a la abundancia de las especies (Clarke \& Warwick, 2001). Se efectuaron mapas de distribución de dichos grupos y de las abundancias de fitoplancton mediante el programa Surfer 8.0. Para comparar la abundancia de zooplancton entre los grupos de estaciones se utilizó la mediana, por ser un valor que representa mejor la abundancia del grupo. 


\section{Resultados}

Hidrografía

En el noroeste del estrecho, predomino la masa de agua del mar de Bellingshausen, allí las temperaturas fueron $>1{ }^{\circ} \mathrm{C}$ y las salinidades $<34.2$ ups (Mar de Bellingshausen Salinidad $<34.2$ ups. y $\mathrm{T}^{\circ} \mathrm{C}>1^{\circ} \mathrm{C}$ ). Al sur este del estrecho, próximo a la Península Antarctica, se presentaron temperaturas más bajas y salinidades más altas, debido a la influencia de la masa de agua del mar de Weddell (Mar de Weddell

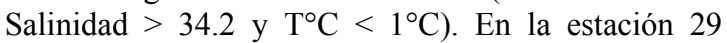
(Figura 1) la temperatura también fue típica del mar de Weddell, sin embargo la salinidad fue menor a 34.2 ups. Esta baja en la salinidad pudo ser por el derretimiento de los hielos que normalmente se producen en dicha zona. Finalmente en el centro del estrecho se observó una mezcla entre estas dos masas de agua (Figueiras et al. 1998; Tenorio, 2006).

Abundancia y distribución del fitoplancton

La abundancia de fitoplancton en la columna de 0 a 100 metros estuvo entre 10106629 y 386772627 cel/L. Las más altas densidades se encontraron al sureste de las islas Shetland del Sur, frente a la Isla Elefante y en el centro del estrecho. Las más bajas en las estaciones próximas a la Península Antártica y al mar de Weddell (Figura 2).

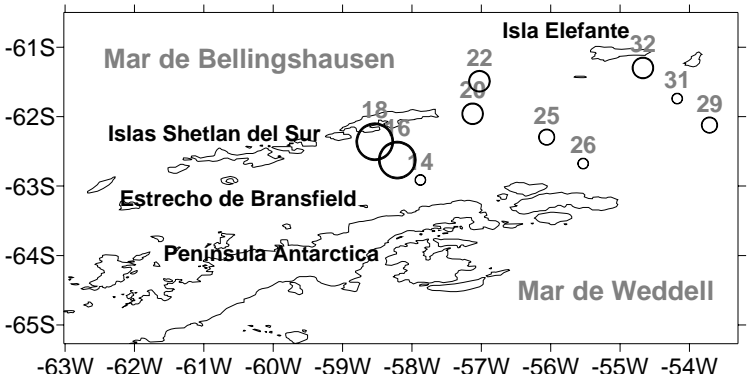

$$
\begin{aligned}
& \circ 10000000-20000000 \mathrm{cel} / \mathrm{L} \text {; } \\
& \circ 20000000-50000000 \mathrm{cel} / \mathrm{L} \text {; } \\
& \text { } 50000000-100000000 \mathrm{cel} / \mathrm{L} \text {; }
\end{aligned}
$$

Figura 2. Distribución de la abundancia total de

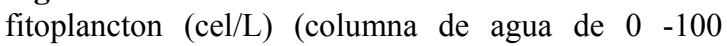
metros) en la zona de estudio.

Se observó una composición muy similar en toda la zona de estudio, compuesta principalmente por microflagelados, los que representaron el $73 \%$ de todo el fitoplancton. Las diatomeas y los dinoflagelados representaron el $21 \%$ y $5 \%$ del fitoplancton respectivamente. Asimismo se encontraron silicoflagelados en las estaciones 14.29 y 31 con bajas abundancias (Abundancia promedio $=534 \mathrm{cel} / \mathrm{L}$ ). Los microflagelados constituyeron más del $60 \%$ del fitoplancton en la mayoría de estaciones a excepción de las estaciones 26 y 32 (Figura 3 ).

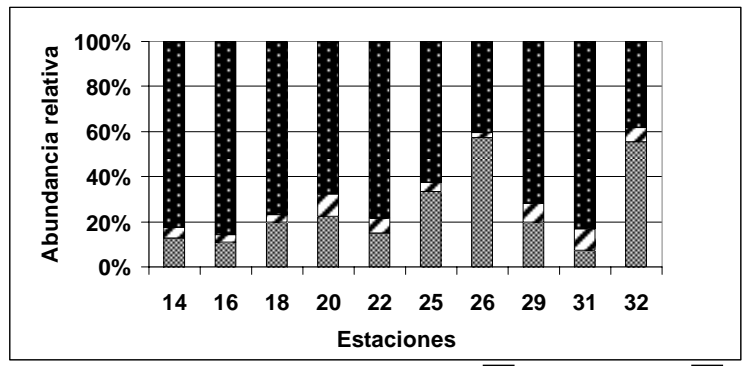

Figura 3. Abundancia relativa de Diatomeas, $\mathbb{Z}$ Dinoflagelados y Microflagelados en las estaciones de muestreo.

\section{Abundancia y distribución del zooplancton.}

La comunidad estuvo compuesta principalmente por copépodos, los que conformaron el $86 \%$ de todo el zooplancton, seguido por los eufáusidos (Abundancia relativa $=4 \%$ ) y quetognatos (Abundancia relativa $=$ $3 \%$ ). Los grupos mas frecuentes fueron los copépodos $($ Frecuencia $=100 \%)$, Eufáusidos (Frecuencia $=$ 100\%), Gasterópodos (Frecuencia $=100 \%$ ) y Quetognatos (Frecuencia $=81 \%$ ). Las salpas fueron poco frecuentes (Frecuencia $=25 \%$ ) y poco abundantes (Abundancia $=28 \mathrm{ind} / 1000 \mathrm{~m}^{3}$ )

En general la composición específica resultó muy similar entre las estaciones de muestreo, siendo las especies más abundantes los copépodos Metridia gerlachei Giesbrecht 1902 y Ctenocalanus sp. Giesbrecht 1888. La primera presentó sus mayores abundancias en las estaciones 16, 25 y 32, y la segunda en las estaciones 22, 31 y 32. En dichas estaciones se registraron las mayores abundancias de zooplancton (Figura 4). Las especies mas frecuentes fueron los copépodos Calanoides acutus Giesbrecht 1902 y Calanus propinquus Brady 1883, encontrándose en el 100\% de las estaciones.

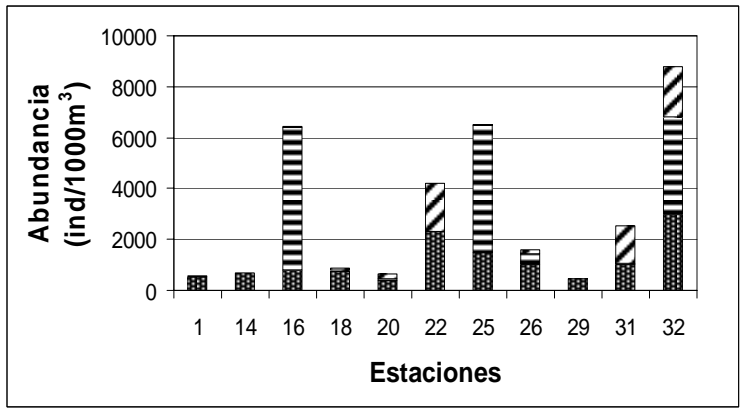

Figura 4. Abundancia de 目 Metridia gerlachei $\square$ Ctenocalanus sp. y de 㹃 otras especies del zooplancton en las estaciones de muestreo.

En el análisis de conglomerados a una distancia de 0.6 se observaron 5 clusters. Dos de ellos contuvieron una estación de muestreo cada uno. Los otros tres agruparon cuatro (Grupo I), dos (Grupo II) y tres (Grupo III) estaciones de muestreo (Figura 5a) El análisis ANOSIM indicó que las diferencias fueron 
significativas entre los grupos (ANOSIM Global $\mathrm{R}=0,858 ; \mathrm{p}<0.001)$.

El Grupo I estuvo conformado por las estaciones del sureste del estrecho y de la Isla Elefante (Estaciones 25, 26, 31 y 32) ubicadas en la masa de agua del mar de Weddell (Figura 5). Allí se encontraron las abundancias más altas de zooplancton $\left(\right.$ Mediana $\left.=4787 \mathrm{ind} / 1000 \mathrm{~m}^{3}\right)$, principalmente por $M$. gerlachei en las estaciones 25 y 32 y de Ctenocalanus sp. en las estaciones 31 y 32 . Asimismo se registraron las especies C. acutus (Mediana $=258 \mathrm{ind} / 1000 \mathrm{~m}^{3}$ ) y C. propinquus (Mediana $=211 \mathrm{ind} / 1000 \mathrm{~m}^{3}$ ) con abundancias altas, y fueron las únicas estaciones en donde se registró al copépodo Oithona frigida Giesbrecht $1902\left(\right.$ Mediana $\left.=181 \mathrm{ind} / 1000 \mathrm{~m}^{3}\right)$.

El Grupo II agrupó las estaciones 16 y 20 (Figura 5 ), ubicadas en el centro del estrecho, en donde se encontró el Frente de Bransfield. En dichas estaciones se presentaron las más bajas abundancias de los copépodos C. propinquus (Mediana $=65 \mathrm{ind} / 1000 \mathrm{~m}^{3}$ ) y C. acutus (Mediana $=34$ ind $/ 1000 \mathrm{~m}^{3}$ ). En la estación 16 se obtuvieron abundancias muy altas de zooplancton (6 $532 \mathrm{ind} / 1000 \mathrm{~m}^{3}$ ), debido a la alta abundancia de $M$. gerlachei (5 595 ind/1000 $\mathrm{m}^{3}$ ) (Figura 4).

El Grupo III, estuvo conformado por las estaciones ubicadas en el noroeste del estrecho (Estaciones 1, 18 y 22), próximas a las islas Shetland del Sur, influenciadas por las masas de agua del mar de Belllingshausen (Figura 5). El copépodo $C$. propinquus (Mediana $=235 \mathrm{ind} / 1000 \mathrm{~m}^{3}$ ) fue una de las especies que mas contribuyó a que estas estaciones se agrupen, ya que presentó abundancias altas y muy similares entre las estaciones. Dentro de este grupo se mostraron bajas abundancias de zooplancton en las estaciones 1 y 18; sin embargo, en la estación 22 las abundancias resultaron altas, especialmente por la presencia de los copépodos Ctenocalanus sp. (Abundancia $=1929$ ind $/ 1000 \mathrm{~m}^{3}$ ) C. propinquus Brady 1883 (Abundancia $=455$ ind/1000 $\left.\mathrm{m}^{3}\right), \quad$ C. acutus Giesbrecht, 1902 (Abundancia $=573$ ind $/ 1000 \mathrm{~m}^{3}$ ), Rhincalanus gigas Brady 1883 (Abundancia $=449$ ind $/ 1000 \mathrm{~m}^{3}$ ); el quetognato Eukrohnia hamata Thiel 1938 (Abundancia $=480$ ind $/ 1000 \mathrm{~m}^{3}$ ) y los estadios tempranos (Caliptopis) de Euphausia spp. Dana 1852 (Abundancia $=284 \mathrm{ind} / 1000 \mathrm{~m}^{3}$ ).

Finalmente las estaciones 14 y 29, correspondientes cada una a un cluster individual, distinguiéndose ambos de los otros tres clusters, estuvieron ubicadas en la masa de agua del mar de Weddell (Figura 5b) y mostraron las más bajas abundancias (Mediana $=670 \mathrm{ind} / 1000 \mathrm{~m}^{3}$ ) y número de especies. C. propinquus Brady 1883 (Mediana = 286 ind $/ 1000 \mathrm{~m}^{3}$ ) fue la más abundante en esas estaciones y Oithona similis Claus 1866 presentó su mayor abundancia en la estación 29 (Abundancia $=96$ ind $/ 1000 \mathrm{~m}^{3}$ ).
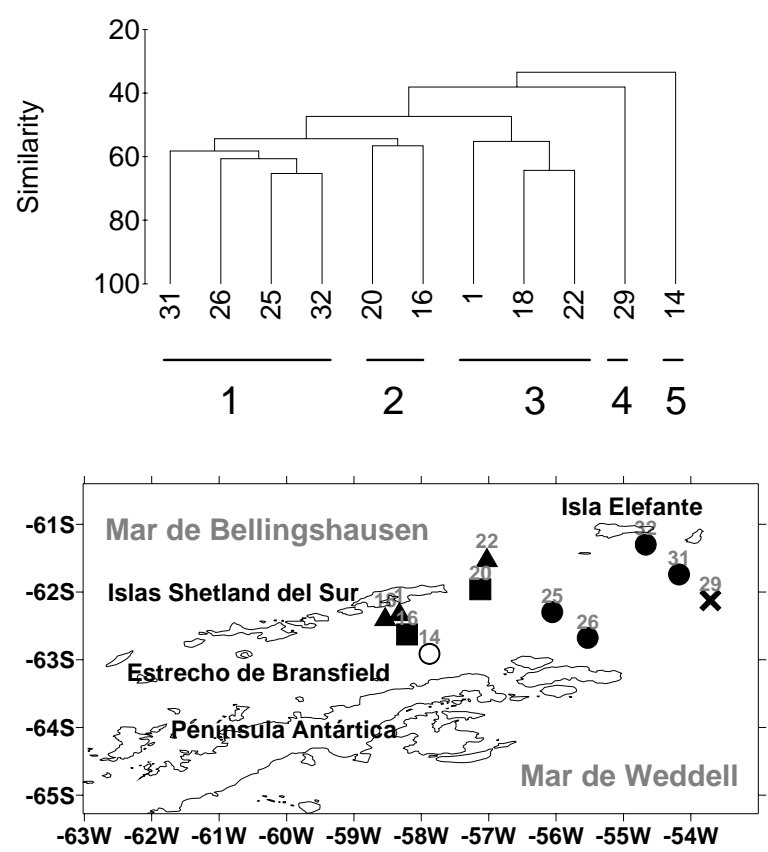

Figura 5. a) Análisis de conglomerados Cluster de las estaciones y b) Distribución de los grupos de estaciones obtenidos del análisis Cluster (•Grupo 1 -Grupo $2 \boldsymbol{\Delta}$ Grupo $3 \times$ estaciones que no fueron agrupadas en el análisis Cluster).

\section{Discusión}

Las masas de agua de los mares de Weddell y de Bellingshausen tuvieron una gran influencia en la biogeografía de las comunidades zooplanctónicas. Por un lado en la masa de agua del mar de Weddell, distribuida al sureste del estrecho y de la Isla Elefante, se encontró un grupo de estaciones caracterizado por presentar la mas alta abundancia de zooplancton y una comunidad compuesta principalmente por copépodos fitófagos, los que pudieron ejercer un intenso pastoreo y dar origen a la baja abundancia de fitoplancton encontrada allí. Sin embargo en las estaciones 32 (perteneciente a este grupo) y 22 (perteneciente al grupo III) a pesar de la gran abundancia de zooplancton registrada el fitoplancton fue muy abundante. Esto sugiere que allí el pastoreo fue menos intenso. Lo cual puede deberse a muchos factores, por un lado se ha demostrado que no solo es importante la cantidad del alimento disponible si no también la cualidad que este tenga para que estas especies se alimenten de una manera óptima (Schnack, 1983; Atkinson, 1995; Pasternak \& Schnack-Schiel, 2007). También se ha demostrado que la temperatura afecta la tasa alimenticia de algunas especies zooplanctónicas (Castellani et al., 2005; Sun et al., 2004), sin embargo es muy difícil afirmar porque ocurrió esto en este estudio, al no existir información suficiente, en especial sobre consumo de alimento. 
Otro grupo de estaciones (Grupo III) se distribuyó al Noroeste del estrecho, influenciado por la masa de agua del mar de Bellingshausen. Allí Gutiérrez et al. (2006), mediante análisis acústicos, registraron una alta concentración de E. superba (krill). Entonces, si esta especie se alimenta mayormente de fitoplancton (Atkinson \& Zinder, 1997) ¿Por que las concentraciones de fitoplancton fueron altas en esa zona? Si bien es cierto que la información no es suficiente para afirmar que sucedió, es posible que la presencia de E. superba fuera reciente, atraída por el gran alimento disponible. Otra explicación pudo ser que esta especie, al tener un carácter omnívoro (Atkinson \& Zinder, 1997), consumió más copépodos que fitoplancton, lo cual también explicaría la baja abundancia de copépodos encontrada allí.

Finalmente otro grupo (Grupo II) se ubicó en el frente de Bransfield, allí se presentaron altas concentraciones de fitoplancton, sin embargo la abundancia de la mayoría de especies zooplanctónicas fue baja, a excepción de $M$. gerlachei. Esto sugiere que la fuerte turbulencia causada por el frente (Tenorio, 2006) dio lugar a un ambiente poco propicio para la mayoría de especies, sin embargo la extensa migración vertical que posee $M$. gerlachei, le pudo permitir migrar a mayor profundidad y resistir la fuerte turbulencia producida en la superficie (Hopkins \& Torres, 1988; Huntley \& Escritor, 1992; Atkinson et al., 1996; Ward \& Shreeve, 1999).

En las estaciones 14 y 29 no obstante que el fitoplancton fue escaso $C$. propinquus obtuvo abundancias altas, probablemente por ser una especie extremadamente oportunista que se alimenta de lo que haya disponible incluyendo partículas, flagelados $\mathrm{u}$ otras algas (Petersen et al., 1999). Este carácter pudo haberle dado la oportunidad de encontrarse en toda la zona de estudio. $O$. similis también adquirió su mayor abundancia en la estación 29, a pesar del escaso alimento encontrado allí. Se ha demostrado que esta especie presenta una tasa de respiración mas baja que otros copépodos (Castellani et al., 2005), por lo tanto su requerimiento energético es mucho menor, es por eso que es capaz de sobrevivir en ambientes donde los recursos alimenticios son limitados, como se observó en este estudio.

\section{Conclusiones}

- La comunidad zooplanctónica estuvo compuesta principalmente por copépodos fitófagos, siendo Metridia gerlachei y Ctenocalanus sp. las especies más abundantes y las que determinaron las altas abundancias encontradas en el centro del estrecho y al sureste de la isla elefante.

- Las masas de agua de los mares de Weddell, y de Bellingshausen, ubicas al sureste y al noroeste del estrecho de Bransfield respectivamente, influyeron en la biogeografía de las diferentes comunidades zooplanctónicas encontradas.
- A pesar de la gran oferta alimenticia encontrada en el frente de Bransfield, la mayoría de especies zooplanctónicas presentaron bajas abundancias, probablemente por las condiciones desfavorables causadas por las fuertes turbulencias del frente. Sin embargo Metridia gerlachei obtuvo sus mayores abundancias allí, pudiendo ser por la fuerte migración vertical que realiza.

\section{Agradecimientos}

Este estudio fue realizado dentro del proyecto COPEPOD I financiado por el Instituto Antártico Peruano (INANPE) y el Instituto del Mar del Perú (IMARPE).

\section{Literatura citada}

Atkinson A., Ward P., Williams R. \& Poulet S.A. 1992. Feeding rates and diel vertical migration of copepods near South Georgia: comparison of shelf and oceanic sites. Marine Biology. 114: 49-56.

Atkinson A. 1995. Omnivory and feeding selectivity in five copepod species during spring in the Bellingshausen Sea, Antarctica. ICES Journal of Marine Science: Journal du Conseil. 52: 385-396.

Atkinson A., Shreeve R.S., Pakhomov E.A., Priddle J., Blight S.P. \& Ward P. 1996. Zooplankton response to a phytoplankton bloom near South Georgia, Antarctica. Mar Ecol Prog Ser. 144: 19.5-210.

Atkinson A. \& Zinder R. 1997. Krill-copepod interactions at South Georgia, Antarctica, I. Omnivory by Euphausia superba. Mar Ecol Prog Ser. 160: 63-73.

Atkinson A., Ward P., Hill A., Brierley A.S. \& Cripps G.C. 1999. Krill-copepod interactions at South Georgia, Antarctica, 11. Euphausia superba as a major control on copepod abundance. Mar Ecol Prog Ser. 176: 63-79.

Bargu1 S., Powell C.L., Coale S.L., Busman M., Doucette G.J. \& Silver M.W. 2002. Krill: a potential vector for domoic acid in marine food webs. Mar Ecol Prog Ser. 237: 209-216.

Brinton E. 1991. Distribution and population structures of immature and adult Euphausia superba in the western Bransfield Strait region during the 1986-87 summer. Deep-Sea Research. 38: 1169-1194.

Castellani C., Robinson C., Smith T. \& Lampi R.S. 2005. Temperature affects respiration rate of Oithona similis. Mar Ecol Prog Ser. 285: 129-135.

Clarke K.R. \& Warwick R.M. 2001. Communities: An Approach to Statistical Analysis and Interpretation PRIMER-E. Segunda Edición. Plymouth, United Kingdom.

Figueiras F.G., Estrada M., López O. \& Arbones B. 1998. Photosynthetic parameters and primary production in the Bransfield Strait: relationships with mesoscale hydrographic structures. Journal of Marine Systems. 17: 129-141.

García M.A., López O., Sospedra J., Espino M., Grácia V., Morrison G., Rojas P., Figa J., Puigdefábregas J. \& Arcilla A.S. 1994. Mesoscale variability in the Bransfield Strait region_Antarctica. During Austral summer. Ann. Geophysicae. 12: 856-867.

García M.A., Castro C.G., Rios A.F., Doval M.D., Rosón G., Gomis D. \& López O. 2002. Water masses and distribution of physic o-chemical properties in the 
Western Bransfield Strait and Gerlache Strait during Austral summer 1995/96. Deep-Sea Research. 49: 585602.

Gutiérrez M., Peraltillo S., Bertrand A., Castillo R., Gonzáles A. \& Swartzman G. 2006. Biología y ecología de las principales comunidades pelágicas del estrecho de Bransfield y alrededor de la isla elefante. Informe anual del proyecto COPEPOD I.

Hopkins T.L. \& Torres J.J. 1988. The zooplankton community in the vicinity of the ice edge, western Weddell Sea, March 1986. Polar Biology. 9: 79-87.

Hubold G. \& Ekau W. 1990. Feeding patterns of post-larval and juvenile notothenioids in the southern Weddell Sea (Antarctica). Polar Biology. 10: 255-260.

Huntley M.E., Brinton E., López M.D.G., Townsend A. \& Nordhausen W. 1990. RACER: Fine-scale and mesoscale zooplankton studies during the spring bloom, 1989. Antarctic Journal of the United States. 25: 157159.

Huntley M.E. \& Escritor F. 1992. Ecology of Metridia gerlachei Giesbrecht in the western Bransfield Strait, Antarctica Deep Sea Research Part A. Oceanographic Research Papers. 39: 1027-1055.

López O., García M.A., Gomis D., Rojas P., Sospedra J. \& Arcilla A.S. 1999. Hydrographic and hydrodynamic characteristics of the eastern basin of the Bransfield Strait (Antarctica). Deep-Sea Research. 46: 1755-1778.

Niiler P.P., Amos A. \& Hu J.H., 1991. Water masses and $200 \mathrm{~m}$ relative geostrophic circulation in the western Bransfield Strait region. Deep-Sea Res. 38: 943-959.

Pasternak A.F. \& Schnack-Schiel S.B. 2007. Feeding of Ctenocalanus citer in the eastern Weddell Sea: low in summer and spring, high in autumn and winter. Polar Biology. 30: 493-501.

Petersen S.F., Sargent J.R., Lønne O.J. \& Timofeev S. 1999. Functional biodiversity of lipids in Antarctic zooplankton: Calanoides acutus, Calanus propinquus, Thysanoessa macrura and Euphausia crystallorophias. Polar Biology. 21: 37-47.

Quetin L.B. \& Ross R.M. 1991. Behavioral and Physiological Characteristics of the Antarctic Krill, Euphausia superba. American Zoologist. 31: 49-63.
Silva N.S., Helbling E.W., Villafa V.E., Amos A.F. \& Holm-Hansen O. 1995. Variability in nutrient concentrations around Elephant Island, Antarctica, during 1991-1993. Polar research. 14: 69-82.

Schnack S. B. 1983. Feeding of two Antarctic copepod species (Calanus propinquus and Metridia gerlachei) on a mixture of centric diatoms. Polar Biology. 2: 63-68.

Sun C., Li S., Wang R. \& Wang X. 2004. Feeding and respiration rates of a planktonic copepod (Calanus sinicus) oversummering in Yellow Sea Cold Bottom Waters. 2004. Marine Biology. 145: 149-157.

Takahashi A., Dunn1 M.J., Trathan P.N., Sato K., Naito Y. \& Croxall J.P. 2003. Foraging strategies of chinstrap penguins at Signy Island, Antarctica: importance of benthic feeding on Antarctic krill. Mar Ecol Prog Ser. 250: 279-289.

Tenorio J. 2006. Resultados condiciones físicas oceanográficas en la expedición ANTAR XVI. Informe anual del proyecto COPEPOD I.

Tokarczyk R. 1987. Classification of water masses in the Bransfield Strait and southern part of the Drake Passage using a method of statistical multidimensional analysis. Pol. Polar. Res. 8: 333-366.

Ward P. \& Shreeve R.S. 1999. The spring mesozooplancton community at South Georgia: a comparison of shelf and oceanic sites. Polar Biol. 22: 289-301.

Ward P., Shreeve R.S., Whitehouse M., Korb B., Atkinson A., Meredith M., Pond D., Watkins J., Goss C. \& Cunningham N. 2005. Phyto- and zooplankton community structure and production around South Georgia (Southern Ocean) during Summer 2001/02. Deep-Sea Research. 52: 421-441.

Zhou M., Nordhausen W. \& Huntley M.E. 1994. ADCP measurements of the distribution and abundance of Euphausiids near the Antarctic Peninsula in winter. Deep- Sea Research. 41: 1425-1445.

\footnotetext{
${ }^{1}$ Jessica Bonicelli, Área de Evaluación de Producción Secundaria del Instituto del Mar del Perú (IMARPE), Esquina Gamarra y General Valle S/N Chucuito Callao 5 - Perú. jecabonicelli@yahoo.com

${ }^{2}$ Diana López, P, Departamento de Limnología, Museo de Historia Natural de la Universidad Nacional Mayor de San Marcos, Av. Arenales 1256 Jesús Maria Lima14 - Perú. dmabelp@gmail.com

${ }^{3}$ Noemi Ochoa, L, Laboratorio de Ecología acuática, Universidad Nacional Mayor de San Marcos Av. Venezuela Cdra. 34 / Av. Universitaria, Lima 1 - Perú. aochoal@unmsm.edu.pe

${ }^{4}$ Rachael Shreeve, British Antarctic Survey High Cross Madingley Road Cambridge CB3 0ET - United Kingdom.rssh@bas.ac.uk
} 